\title{
Rotavírus $A$ em crianças de até três anos de idade, hospitalizadas com gastroenterite aguda em Campo Grande, Estado do Mato Grosso do Sul
}

\author{
Rotavirus $A$ among hospitalized infants, up to three years of age, \\ with acute gastroenteritis in Campo Grande, \\ State of Mato Grosso do Sul
}

\author{
Márcia Sueli Assis Andreasii ${ }^{1}$, Sonia Maria Fernandes Batista ${ }^{1}$, Inês Aparecida Tozetti, \\ Cláudia Okanobo Ozaki ${ }^{1}$, Mariana Menegusso Nogueira ${ }^{1}$, Fabíola Souza Fiaccadori ${ }^{2}$, \\ Ana Maria Tavares Borges ${ }^{2}$, Rodrigo Alessandro Togo Santos ${ }^{2}$ \\ e Divina das Dores de Paula Cardoso ${ }^{2}$
}

\begin{abstract}
RESUMO
Através da eletroforese em gel de poliacrilamida e do ensaio imunenzimático combinado para rotavírus e adenovirus, foram analisadas 380 amostras fecais de crianças com até 3 anos, hospitalizadas com diarréia aguda, entre maio de 2000 e janeiro de 2004, em Campo Grande, MS. Do total de amostras, 88 (23,2\%) foram positivas para Rotavirus A. Dentre essas, 81 (92\%) tiveram padrão eletroferotípico definido, sendo 77 (87,5\%) de padrão longo e quatro (4,5\%) de padrão curto. A caracterização genotípica G e P foi feita por RT-Nested-PCR para 85 amostras, sendo 56 (65,9\%) genotipáveis para genótipo G. Dentre essas, 49 (87,5\%) foram G1, cinco (8,9\%) G4, uma (1,8\%) G3 e uma (1,8\%) G9. Considerando a genotipagem P, 37 (43,5\%) foram genotipáveis e todas eram P[8]. A associação G e P mais observada foi G1P [8], 33 (89,2\%) amostras; seguida de G4P[8], duas (5,4\%) amostras; G3P[8], uma (2,7\%) amostra; e G9P[8], uma (2,7\%) amostra.
\end{abstract}

Palavras-chaves: Rotavirus A. Infecção. Criança. Genotipagem.

\section{ABSTRACT}

Polyacrylamide gel electrophoresis and combined immunoenzyme assay for rotavirus and adenovirus were used to analyze 380 fecal samples from children up to three years of age who were hospitalized with acute diarrhea in Campo Grande, State of Mato Grosso do Sul, between May 2000 and Jamuary 2004. Among all the samples, 88 (23.2\%) were positive for Rotavirus A. Out of these, 81 (92\%) had a defined electrophoretic pattern: 77 (87.5\%) with a long pattern and four (4.5\%) with a short pattern. Genotype G and P characterization was done by nested RT-PCR for 85 samples, of which 56 (65.9\%) were genotyped as type G. Among these, 49 (87.5\%) were G1, five (8.9\%) were G4, one (1.8\%) was G3 and one (1.8\%) was G9. The genotype was found to be type P in 37 samples (43.5\%) and all of these were P [8]. The G and P association most observed was G1P[8], with 33 samples (89.2\%), followed by G4P [8], two samples (5.4\%); G3P[8], one sample (2.7\%); and G9P[8], one sample (2.7\%).

Key-words: Rotavirus A. Infection. Children. Genotyping.

Os Rotavírus A são considerados, em escala universal, importantes agentes etiológicos de diarréia aguda em bebês e crianças menores de 3 anos de idade e uma das causas mais comuns de hospitalização de crianças na faixa etária de zero a quatro anos ${ }^{27}$.

As partículas virais completas apresentam $75 \mathrm{~nm}$ de diâmetro, morfologia esférica, simetria icosaédria, sem envelope lipídico.
O capsídio viral é organizado em três camadas concêntricas de proteínas que envolvem o genoma, composto de 11 segmentos de RNA de dupla fita (dsRNA) ${ }^{20}$.

De acordo com variações nos segmentos genômicos codificantes das proteínas VP7 e VP4, formadoras do capsídeo externo, os Rotavírus A apresentam diferentes genótipos G (15) e P (20), respectivamente, com predomínio, em escala universal,

\footnotetext{
1. Departamento de Patologia, Universidade Federal de Mato Grosso do Sul, Campo Grande, MS. 2. Instituto de Patologia Tropical e Saúde Pública, Universidade Federal de Goiás, Goiânia, GO.

Endereço para correspondência: Drª Márcia S.A. Andreasi. Dept ${ }^{\circ}$ de Patologia/UFMS. Cidade Universitária s/n, 79070-900 Campo Grande, MS. Tel: 5567 3345-7387

e-mail:msaa@nin.ufms.br

Recebido em: 21/03/2007

Aceito em: 21/06/2007
} 
do genótipo G1P[8]. No Brasil, os genótipos mais comumente observados são G1P[8], G3P[8], G4P[8], G2P[4], embora outras combinações tenham sido detectadas como G1P[6], G2P [6], G9P [8], o que evidencia uma multiplicidade de amostras circulantes $^{48101517212434}$.

No Brasil, a gastroenterite aguda por Rotavirus $A$ tem sido estimada, considerando os casos de hospitalização, em 12 a $42 \%^{82933}$.

A importância epidemiológica da gastroenterite por Rotavírus $A$ em todo o mundo, com a implantação do processo vacinal, impõe a necessidade de caracterização das amostras virais circulantes em cada região geográfica. Com a finalidade de conhecer a epidemiologia regional e de contribuir para a implementação das medidas de controle e prevenção da gastroenterite por Rotavírus A no Estado de Mato Grosso do Sul, a proposta deste trabalho foi a de determinar a prevalência de Rotavirus A, bem como os genotipos G e P circulantes desse vírus em crianças hospitalizadas com quadro de gastroenterite aguda, na cidade de Campo Grande, MS.

\section{MATERIAL E MÉTODOS}

0 material de estudo constituiu-se de 380 amostras de fezes in natura com aproximadamente $10 \mathrm{ml}$, coletadas de igual número de crianças, 226 do sexo masculino e 154 do feminino, com até três anos de idade e internadas no Setor de Pediatria da Santa Casa de Misericórdia com quadro de gastroenterite aguda, durante o período de maio de 2000 a janeiro de 2004 . A coleta das amostras foi realizada após autorização dos pais ou responsável e do preenchimento de uma ficha epidemiológica.

0 estudo foi aprovado pelo Comitê de Ética em Pesquisa da Universidade Federal de Mato Grosso do Sul (protocolo $\mathrm{N}^{0} 205$ ) e financiado pelo Conselho Nacional de Ciência e Tecnologia-CNPq (Processo no. 520729/99-4).

A detecção de Rotavírus $A$ foi realizada utilizando duas metodologias: eletroforese em gel de poliacrilamida (EGPA) ${ }^{29} \mathrm{com}$ a classificação dos eletroferotipos ${ }^{25}$ e ensaio imunoenzimático combinado para rotavírus e adenovírus (EIERA) ${ }^{28}$.

A caracterização genotípica foi feita para genótipos $\mathrm{G}$ e $\mathrm{P}$ por RT-Nested-PCR. Para o procedimento, o dsRNA viral foi extraído seguindo a descrição da literatura ${ }^{3}$. A primeira e a segunda reação de amplificação para G e P foram feitas seguindo protocolos e iniciadores previamente descritos ${ }^{15} 16$.

A análise estatística dos resultados foi feita através do programa Epi Info 6 versão 6.04: Centers for Disease Control and Prevention (CDC), Atlanta, GA), utilizando os testes qui-quadrado $\left(\mathrm{F}^{2}\right)$ e exato de Fisher, com grau de confiança de $95 \%$.

\section{RESULTADOS}

Foi observado que $88(23,2 \%)$ crianças apresentavam positividade para Rotavirus A, sendo $52(59, \%)$ do sexo masculino e $36(41 \%)$ do sexo feminino $(\mathrm{p}=0,934)$.
A análise da positividade em relação a faixa etária e estação climática mostrou percentual maior de positividade em crianças na faixa etária de 7 a 12 meses e também maior pico de ocorrência viral nos meses de abril a agosto $(p=0,009)$ (Tabela 1$)$. Em Campo Grande, o período do estudo (abril-agosto) correspondeu à estação de seca, com temperatura média de $21,7^{\circ} \mathrm{C}$, índice pluviométrico de $60,3 \mathrm{~mm}^{3} \mathrm{e}$ umidade relativa do ar atmosférico de $63,2 \%$. Setembro-março correspondeu à estação chuvosa, com temperatura média de $24,8^{\circ} \mathrm{C}$, índice pluviométrico de $165,9 \mathrm{~mm}^{3} \mathrm{e}$ umidade relativa do ar atmosférico de $72,4 \%$ (dados fornecidos pelo Setor de Metereologia da Empresa Brasileira de Pesquisa Agropecuária - EMBRAPA).

\begin{tabular}{|c|c|c|c|}
\hline \multirow[t]{2}{*}{ Variáveis } & Amostras & Positividade & $\mathrm{p}$ \\
\hline & $\mathrm{n}^{\mathbf{0}}$ & $\%$ & \\
\hline \multicolumn{4}{|l|}{ Faixa etária (meses) } \\
\hline 0 a 6 & 121 & 15,7 & 0,009 \\
\hline 7 a 12 & 147 & 30,6 & \\
\hline 13 a 24 & 103 & 22,3 & \\
\hline 25 a 36 & 9 & 11,1 & \\
\hline \multicolumn{4}{|l|}{ Estação climática } \\
\hline seca (abril /agosto) & 147 & 30,6 & 0,009 \\
\hline chuvosa (setembro/março) & 121 & 15,7 & \\
\hline
\end{tabular}

Das 82 amostras positivas pela EGPA, 81 (98,7\%) tiveram 0 padrão eletroferotípico definido, sendo 77 (95,1\% ) de padrão longo e 4 ( $4,9 \%$ ) de padrão curto.

Considerando a genotipagem, das 85 amostras analisadas, $56(65,8 \%)$ foram genotipáveis e, dentre essas, 49 (87,5\%) foram classificadas como G1, cinco $(8,9 \%)$ como G4, uma $(1,8 \%)$ como G3 e uma (1,8\%) como G9. A genotipagem P mostrou que todas as 37 amostras genotipáveis eram $\mathrm{P}[8]$. A associação $\mathrm{G}$ e P mais comum, com 33 (89,2\%) amostras, foi G1P>@ @seguida de duas

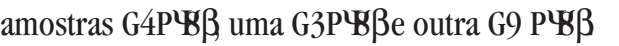

A análise da circulação dos genótipos em relação ao ano de detecção mostrou que G1 ocorreu em todos os anos com exceção de 2001, ano em que foram detectadas as cinco amostras G4. Os genótipos G3 e G9 ocorreram no ano de 2003.

\section{DISCUSSÃo}

Estimativas oriundas de múltiplas investigações realizadas no âmbito hospitalar e ambulatorial em vários países revelam percentuais de positividade para rotavírus que variam de 12 a $50 \% 189101316222634$.

No presente estudo, observou-se índice de positividade de $23,2 \%$ para Rotavirus $A$ e, em estudos realizados anteriormente na região Centro-Oeste do Brasil, os índices de detecção variaram de 11,8\% a 37,2\% em Goiânia ${ }^{810}$, sendo observado índice de $26 \%$ em Brasília ${ }^{4}$. Dessa forma, os resultados deste estudo são concordantes com os da região Centro-Oeste do Brasil, bem como com os de outras partes do mundo. 
Quando se considera a positividade para Rotavírus A em relação a gênero, observa-se que estes dados estão de acordo com o resultado obtido por outros autores, que também não observaram diferença significativa para os gêneros ${ }^{3135}$. Por outro lado, alguns trabalhos mostraram maior índice de positividade para 0 gênero masculino $0^{812}$.

Em nosso estudo foi observado que a infecção por Rotavirus $A$ ocorreu em todas as faixas etárias, incluindo crianças com menos de seis meses de idade, embora com ocorrência maior nas crianças situadas na faixa etária de 7 a 12 meses. A infecção por Rotavirus $A$ na faixa etária de 0 a 6 meses tem sido considerada de ocorrência assintomática ${ }^{23}$, o que contrasta com o observado no presente estudo. Outros estudos, incluindo os realizados em Goiânia ${ }^{568}$, mostram importante índice de infecção sintomática nessa faixa etária, o que pode refletir as condições sócioeconômico-sanitárias da população. Por outro lado, um índice menor de infecção foi observado nas crianças maiores de 24 meses de idade, o que reforça o conceito de que, após os dois anos de vida, o número de casos de infecção por rotavírus tende a diminuir, talvez devido a fato de que grande parte das crianças nessa faixa etária já tenham tido contato com 0 agente, 0 que lhes confere imunidade ${ }^{1937}$.

Neste estudo, foi observado que $95 \%$ das amostras eram padrão longo. Esse resultado está de acordo com outros da literatura, em que se observa uma nítida preponderância do padrão longo em relação ao curto ${ }^{923}$. Também, foi observada uma extensa variabilidade de eletroferotipos, com a conotação ainda de que eram bastante particulares em relação ao ano de ocorrência. Esse fato reforça a opinião de que o padrão de bandeamento dos 11 diferentes segmentos de dsRNA de Rotavirus $A$ fornecem importante informação inicial em termos de variação de amostras em determinado tempo e localidade ${ }^{1122}$.

Neste estudo, observou-se o predomínio do genótipo G1, seguido do G4, G3 e G9. Os genótipos G1-G4 são considerados usuais $^{4714}$, embora outros tipos G como o G5, G8, G9, G10, G11 e G12 tenham também sido detectados ${ }^{173436}$. Esses dados apontam para a possibilidade de mudanças na prevalência de diferentes genótipos por região. A análise em relação ao ano de detecção mostrou que, neste estudo, o genótipo G1 ocorreu em todos os anos com exceção de 2001, quando foram detectados todos os G4. Os genótipos G3 e G9 ocorreram em 2003. As investigações indicam, via de regra, que um determinado genótipo predomina durante um ou dois anos, emergindo, a partir de então, uma nova variedade antigênica dominante ${ }^{18} 233$, o que também implica cuidado relativo a estratégias vacinais ${ }^{232}$. Por outro lado, considerando a genotipagem $P$, todas as amostras genotipáveis foram $\mathrm{P}[8]$ e, como resultado, a associação $\mathrm{G}$ e $\mathrm{P}$ predominante foi G1P[8], o que corrobora estudos de diferentes partes do mundo $0^{8173336}$.

Como um todo, a prevalência de Rotavirus $A$ observada em nosso estudo vem corroborar os dados de outras regiões do mundo, incluindo o Brasil e a região Centro-Oeste. Assim sendo, esses dados podem servir de subsídio no sentido de reforçar as informações concernentes à problemática sócio-econômica decorrente da enfermidade por esses vírus, como o aumento na demanda dos serviços de saúde e a necessidade de hospitalização, o que, inclusive, expõe a criança a outros patógenos, acarreta custos e perda de dias de trabalho dos pais ou responsáveis. 0 estudo aponta, ainda, para a necessidade de contínua monitoração desses vírus dentro da possibilidade da emergência de novas amostras que podem não estar inclusas nas vacinas em uso.

\section{REFERÊNCIAS}

1. Araújo IT, Ferreira MSR, Fialho AM, Assis RM, Cruz CM, Rocha M, Leite JPG. Rotavirus genotypes P [4] G9, P[6] G9, and P[8] G9 in hospitalized children with acute gastroenteritis in Rio de Janeiro, Brazil. Journal of Clinical Microbiology 39:1999-2001, 2001.

2. Bànyai KJR, Gentsch JR, Glass RI, Uj M, Mihàly I, Szuïcs G. Eighty-year survey of human rotavirus strains demonstrates circulation of unusual $\mathrm{G}$ and $\mathrm{P}$ types in Hungary. Journal of Clinical Microbiology 42:393-397, 2004.

3. Boom R, Sol CJA, Salimans MMM, Jansen CL, Wertheim-Van PMED, Van Der Noordaa J. Rapid and simple method for purification of nucleic acids. Journal of Clinical Microbiology 28:495-503, 1990.

4. Braga KNL. Caracterização de genótipos G de rotavírus do grupo A circulantes no Distrito Federal. Dissertação de Mestrado, Universidade de Brasília, Brasília, DF, 2006.

5. Cardoso DDP. Rotavírus em crianças de Goiânia, Goiás: caracterização das amostras de rotavírus do grupo A pela sorotipagem, subgrupagem e padrão eletroforético. Tese de Doutorado, Universidade de São Paulo, São Paulo, SP, 1997.

6. Cardoso DDP, Martins RMB, Kitajima EW, Barbosa AJ, Camarota SCT, Azevedo MSP. Rotavirus and adenovirus in children 0-5 years of age with or without gastroenteritis in hospitals from Goiânia-GO, Brazil. Revista do Instituto de Medicina Tropical de São Paulo 34:433-439, 1992.

7. Cardoso DDP, Soares CMA, Azevedo MSP, Leite JP, Munford V, Rácz ML. Serotypes and subgroups of rotavirus isolated from children in central Brazil. Journal of Health Population and Nutrition 18:39-43, 2000.

8. Cardoso DDP, Soares CMA, Souza MBLD, Azevedo MSP, Martins RMB, Queiroz DA0, Brito WMED, Munford V, Rácz ML. Epidemiological features of rotavirus infection in Goiânia, Goiás, Brazil, from 1986 to 2000. Memórias do Instituto Oswaldo Cruz 98:25-29, 2003.

9. Coluchi N, Munford V, Manzur J, Vazquez C, Escobar M, Weber E, Mármol P, Rácz ML. Detection, subgroup specificity, and genotype diversity of Rotavirus strains in children with acute diarrhea in Paraguay. Journal of Clinical Microbiology 40:1709-1714, 2002.

10. Costa PSS, Grisi SJFE, Cardoso DDP, Fiaccadori FS, Souza MBLD, Santos RAT. Manifestações clínicas e epidemiológicas das infecções por Rotavirus A. Pediatria 26:151-158, 2004.

11. Desselberger U. Classical and molecular techniques for the diagnosis of viral gastroenteritis. Clinical and Diagnostic Virology 5:101:109, 1996.

12. Espinoza F, Paniagua M, Hallander H, Hedlund KO, Svensson L. Prevalence and characteristics of severe rotavirus infections in Nicaraguan children. Annals of Tropical Pediatrics 17:25-32, 1997.

13. Fang ZY, Yang H, Qi J, Zhang J, Sun LW, Tang JY, Ma L, Du ZQ, He AH, Xie JP, Lu YY, Ji ZZ, Zhu BQ, Wu HY, Lin SE, Xie HP, Griffin DD, Ivanoff B, Glass RI, Gentsch JR. Diversity of rotavirus strains among children with acute diarrhea in China: 1998-2000 surveillance study. Journal of Clinical Microbiology 40:1875$1878,2002$.

14. Franco MA, Angel J, Greenberg HB. Immunity and correlates of protection for rotavirus vaccines. Vaccine 24:2718-2731, 2006.

15. Gentsch JR, Glass RI, Woods P, Gouvea V, Gorziglia M, Flores J, Das BK, Bhan MK. Identification of group a rotavirus gene 4 types by polymerase chain reaction. Journal of Clinical Microbiology 30:1365-1373, 1992.

16. Gouvea V, Glass RI, Woods P, Taniguchi K, Clark F, Forrester B, Fang ZY. Polymerase chain reaction amplification and typing of rotavirus nucleic acid from stool specimens. Journal of Clinical Microbiology 28: 276-282, 1990. 
17. Iturriza-Gómara M, Isherwood B, Desselberger U, Gray J. Reassortment in vivo: driving force for diversity of human rotavirus strains isolated in the United Kingdom between 1995 and 1996. Journal of Virology 75:369-3705, 2001.

18. Iturriza-Gómara M, Kang G, Mammen A, Jana AK, Abraham M, Desselberger $\mathrm{U}$, Brown D, Gray J. Characterization of G10P[11] rotaviruses causing acute gastroenteritis in neonates and infants in Vellore, India. Journal of Clinical Microbiology 42:2541-2547, 2004.

19. Jiang B, Gentsch JR, Glass RI. The role of serum antibodies in the protection against rotavirus disease: an overview. Clinical Infectious Diseases Chicago 34: 1351-1361, 2002

20. Kapikian AZ, Hoshino Y, Chanock RM. Rotaviruses. In: Knipe DM, Howley PM, Griffin DE, Martin MA, Lamb RA, Roizaman B (eds) Fields virology. Williams \& Wilkins, Philadelphia, p. 1787-1833, 2001

21. Leite JPG, Alfieri AA, Woods PA, Glass RI, Gentsch JR, Rotavirus G and P types circulating in Brazil: characterization by RT-PCR, probe hybridization, and sequence analyses. Archives of Virology 141:2365-2374, 1996.

22. Linhares AC, Bresse JS. Rotavirus vaccines and vaccination in Latin America. Pan American Journal of Public Health 8:305-331, 2000.

23. Linhares AC, Gabbay YB, Freitas RB, Travassos da Rosa ES, Mascarenhas JDP, Loureiro ECB. Longitudinal study of rotavirus infections among children from Belém, Brazil. Epidemiology and Infection 102:129-145, 1989.

24. Linhares AC, Mascarenhas JDP, Gusmão RHP, Gabbay YB, Fialho AM, Leite JP. Neonatal rotavirus infection in Belém, northern Brazil: Nosocomial transmission of a P[6] G2 strain. Journal of Medical Virology 67:418-426, 2002.

25. Lourenço MH, Nicolas JC, Cohen J, Scherrer R, Bricout F. Study of human rotavirus genome by electrophoresis: attempt of classification among strains isolated in France. Annales de L'Institut Pasteur Virologie 132:161-173, 1981.

26. Marie-Cardine A, Gourlain K, Mouterde 0, Castignolles N, Heflet MF, Mallet E, Buffet-Janvresse C. Epidemiology of acute viral gastroenteritis in children hospitalized in Rouen, France. Clinical Infectious Diseases 34:1170-1178, 2002

27. Ministério da Saúde. Secretaria de Vigilância em Saúde, Departamento de Vigilância Epidemiológica. Informe Técnico. Doença diarréica por rotavírus: vigilância epidemiológica e prevenção pela vacina oral de rotavírus humano (VORH). Ministério da Saúde, Brasília, 2006.
28. Pereira HG, Azevedo RS, Leite JPG, Andrade ZP, Castro L A. Combined enzyme immunoassay for rotavirus and adenovírus (EIERA). Journal of Virological Methods 10: 21-28, 1985.

29. Pereira HG, Leite JPG, Azevedo RS, Farias V, Sutmoller FN. Atypical rotavirus detected in a child with gastroenteritis in Rio de Janeiro, Brazil. Memórias do. Instituto Oswaldo Cruz 78:245-250, 1983.

30. Pereira HG, Linhares AC, Candeias JAN, Glass RI. National laboratory surveillance of viral agents of gastroenteritis in Brazil. Bulletin of Pan American Health Organization 27:224-233, 1993.

31. Pérez-Schael I, González R, Fernández R, Alfonso L, Inaty D, Boher Y, Sarmiento L. Epidemiological features of rotavirus infection in Caracas, Venezuela: implications for rotavirus immunization programs. Journal of Medical Virology 59:520-526, 1999.

32. Ramachandran M, Vij A, Kumar R, Das BK, Gentsch JR, Bhan MK, Glass RI. Lack of maternal antibodies to $\mathrm{P}$ serotypes may predispose neonates to infections with unusual rotavirus strains. Clinical and Diagnostic Laboratory Immunology 5:527-530, 1998

33. Santos N, Soares CC, Volotão EM, Albuquerque MCM, Hoshino Y. Surveillance of rotavirus strains in Rio de Janeiro, Brazil, from 1997 to 1999. Journal of Clinical Microbiology 7:3399-3402, 2003.

34. Santos N, Volotão EM, Soares CC, Campos GS, Sardi SI, Hoshino Y. Predominance of rotavirus genotype G9 during the 1999, 2000, and 2002 seasons among hospitalized children in the city of Salvador, Bahia, Brazil: implications for future vaccine strategies. Journal of Clinical Microbiology 8:4064-4069, 2005.

35. Souza MBLD. Caracterização sorológica e molecular de amostras de Rotavirus A proveniente de amostras fecais de crianças com gastroenterite aguda em Goiânia-GO. Dissertação de Mestrado. Universidade Federal de Goiás, Goiás, 2001.

36. Uchida R, Pandey BD, Sherchand JB, Ahmed K, Yokoo M, Nakagomi T, Cuevas LE, Cunliffe NA, Hart CA, Nakagomi O. Molecular epidemiology of rotavirus diarrhea among children and adults in Nepal: detection of G12 strains with $\mathrm{P}$ [6] or P[8] and G11P[25] strain. Journal of Clinical Microbiology 44:3499-3505, 2006.

37. Velasquez FR, Matson DO, Guerrero ML, Shults J, Calva JJ, Morrow AL, Glass RI, Pickering LK, Ruiz-Palacios GM. Serum antibody as a marker of protection against natural rotavirus infection and disease. The Journal of Infections Diseases 182:1602-1609, 2000 